\title{
Snapshot-Based Data Backup Scheme: Open ROW Snapshot ${ }^{\star}$
}

\author{
Jinsun Suk, Moonkyung Kim, Hyun Chul Eom, and Jaechun No \\ Dept. of Computer Software \\ College of Electronics and Information Engineering \\ Sejong University, Seoul, Korea
}

\begin{abstract}
In this paper, we present the design and implementation details of the Open ROW Snapshot which is the data backup scheme based on the snapshot approach. As the data to be stored in storage systems are tremendously increased, data protection techniques have become more important to provide data availability and reliability. Snapshot is one of such data protection techniques and has been adopted to many file systems. However, in large-scale storage systems, adopting a snapshot technique to prevent data loss from intentional/accidental intrusion is not an easy task because the data size being backup-ed at a given time interval may be huge. In this paper, we present the Open ROW Snapshot that has been implemented based on the file system-based snapshot approach. The Open ROW Snapshot provides a widely portable structure and causes less I/O processing overhead than the ROW(Redirect-On Write) method does. Furthermore, the Open ROW Snapshot provides a capability of maintaining the disk space assigned to snapshot images in a consistently-sized disk portion. We present the performance results of the Open ROW Snapshot obtained from the Linux cluster located at Sejong University.
\end{abstract}

\section{Introduction}

Many data recovery approaches 11,12,13 have been developed to protect important data against system crash. Especially, the snapshot-based data recovery has been adopted to many file systems $[5,6,7,8,9] 10]$ to provide data availability and reliability. However, in large-scale storage systems, implementing a snapshot technique to prevent data loss from intentional/accidental intrusion is not an easy task because the data size being backup-ed at a given time interval may be huge. Simply duplicating the inodes and data blocks associated with a pointin-time snapshot causes high I/O processing overhead. Furthermore, maintaining a large number of snapshot images consume a large portion of disk space.

Snapshots can be built in two different ways; one is a volume-based snapshot in which the snapshot images are taken under LVM (Logical Volume Manager), and the other is a file system-based snapshot in which all the snapshot related operations are performed under the file system control. Even though the volume-based

\footnotetext{
^ This work was supported by a Seoul R\&BD program.
} 
snapshot can efficiently manage snapshot images and disk space, it requires to preserve some disk space for retaining snapshot images. The file system-based snapshot does not need to reserve the disk space to store snapshot images. However, because the file system-based snapshot is tightly coupled to the underlying file system, porting a snapshot implementation among several file systems would take considerable overheads. Porting a snapshot becomes even worse when file systems that are supposed to use a snapshot support different block allocation policies.

We developed the Open ROW Snapshot that combines the good features of both file system-based method and ROW approach. Our primary objectives in developing the Open ROW Snapshot were to minimize I/O processing overhead occurred between successive snapshot images, to provide a wide range of portability by supporting both the extent-unit and block-unit allocation policies, and to provide a capability of managing disk space for snapshots in a consistent size of disk section. In order to minimize I/O processing overhead occurred in duplicating inodes and data blocks to take a point-in-time snapshot, we chose to adopt the ROW-based snapshot approach. Besides, we used a pre-allocated metatdata to reduce the block allocation time for each instantaneous snapshot. Furthermore, the Open ROW Snapshot can easily be combined with the extent-based storage structure [12, as well as be combined with the block-based storage structure 34. When the Open ROW Snapshot is combined with an extentbased storage structure, it can easily detect the sharing of data blocks between several snapshot images by checking the value of the bitmap flag. This helps to efficiently eliminate the corrupted snapshot images in the snapshot history.

The rest of this paper is organized as follows. In Section 2, we discuss the design motivations of the Open ROW Snapshot. Section 3 describes the implementation details of the Open ROW Snapshot and in Section 4, we present the performance results obtained from the Linux cluster at Sejong University. In Section 5, we conclude our paper.

\section{Design Motivation}

\subsection{Minimize I/O Processing Time}

In order to minimize I/O processing overhead occurred in duplicating inode and data blocks to take a point-in-time snapshot, we chose to adopt the ROW-based snapshot approach. Additionally, in adopting the ROW approach, we used a pre-allocated metadata to reduce the block allocation time for each instantaneous snapshot. When the inode of an active file is created, we also allocate an additional inode to be used for the following snapshot image. When a point-intime snapshot is taken, this additional inode becomes an inode of the snapshot file. Also, instead of simply duplicating all the associated blocks of the snapshot file, the snapshot inode just links pointers to the original blocks to denote that these data blocks are shared between the active file and the snapshot file. In this way, we can significantly reduce the processing time for the block allocation, compared to that of COW(Copy On Write) approach. 


\subsection{Provide a Wide Range of Portability}

The Open ROW Snapshot can easily be combined with the extent-based storage structure where a contiguous number of blocks are allocated to a file segment, as well as can be combined with the block-based storage structure. When the Open ROW Snapshot is combined with the extent-based structure, such as XFS [12], each inode, including the inode of a snapshot image, contains the extents composed of three components; the starting block address, block count describing the number of blocks contiguously allocated, and bitmap flag describing the sharing of the data blocks belonging to the extent. If the bitmap flag is set to 1 , it would then denote that the data blocks belonging to the corresponding extent are recently allocated, and thus no other inode currently shares these data blocks yet. If the flag is set to 0 , it would then mean that the data blocks belonging to the associated extent are shared between files, thus a careful block management is required while the modification to the data blocks happens to these blocks.

\subsection{Manage Disk Space for Snapshots}

We developed a snapshot spatial algorithm that enables us to keep the disk space allocated for snapshot images as smallest as possible. In the Open ROW Snapshot, all the snapshot images, including their active file, are grouped by two pointers, prev_core_snap and next_core_snap, and in each snapshot group, the active file is linked at the front and the oldest snapshot image is linked at the back. Constructing a snapshot group of an active file enables us to easily trace back and forth the link to find which data blocks are shared between files. When the Open ROW Snapshot finds corrupted or backup-ed snapshot images while traversing a snapshot group, the snapshot spatial algorithm can easily check the sharing of the data blocks belonging to those images. It also unlinks the associated inode from the snapshot group, and thus enables to keep snapshot images in a consistently-sized disk section.

\section{Implementation Details}

\subsection{Overall Structure}

Figure 1 illustrates an overview of the Open ROW Snapshot. In this Figure, the Open ROW Snapshot was configured to support for the extent-based allocation. For two active files File1 and File2, there exist two snapshot files each, snapshot1 and snapshot2. The Open ROW Snapshot uses two pointers, prev_core_snap and next_core_snap, to easily make a snapshot group of an active file, and thus to verify the integrity of each snapshot file with low processing overhead. The file pointed by prev_core_snap is of a preceding snapshot image, while conversely, the file pointed by next_core_snap is of a following snapshot image or an active file.

Figure 1 shows how the snapshot images are grouped as time goes by. At $t 0$, two active files, File1 and File2 are created, and then, at $t 1$, these two 


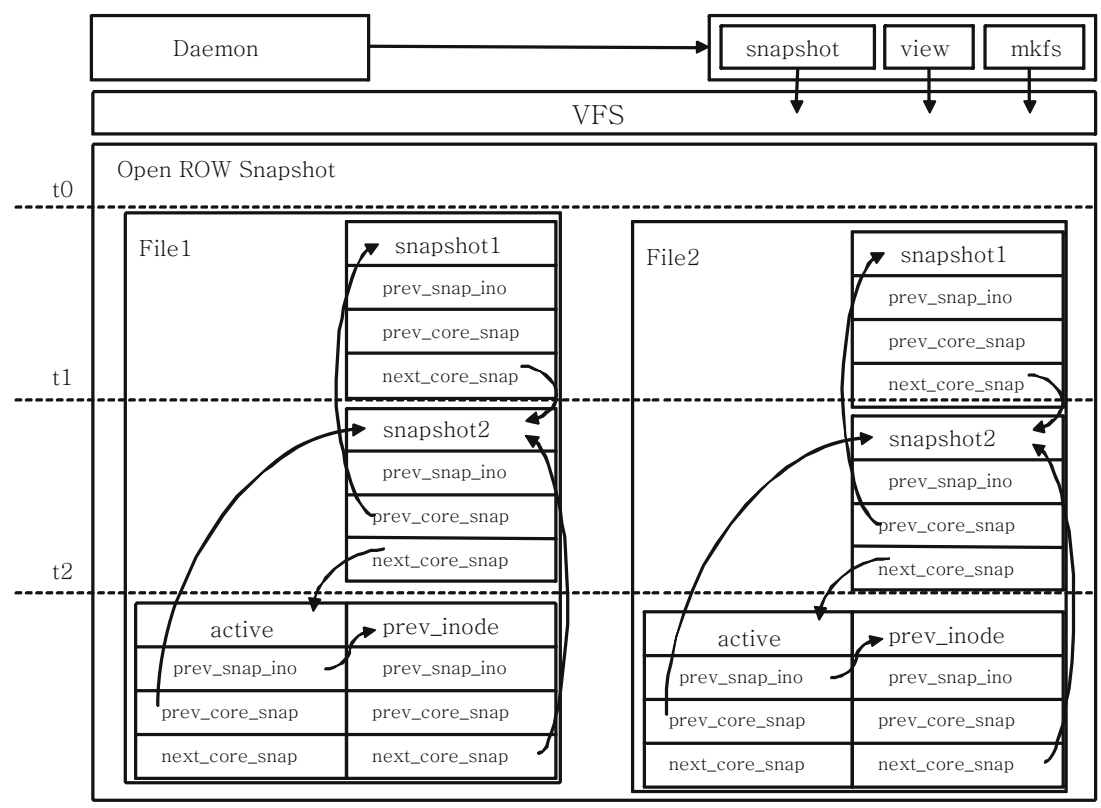

Fig. 1. An overview of the snapshot structure

files became the snapshot images of their active files, while being linked with their active files by using the prev_core_snap. To easily find out which is the next snapshot image of a file, each snapshot image is linked with the following one by using the next_core_snap. Grouping the related snapshot images with these two pointers enables for the Open ROW Snapshot efficiently to perform the snapshot spatial algorithm to eliminate a corrupted snapshot image or a backup-ed snapshot image. As a result, the snapshot files can be stored in a small-sized disk space.

When the inode of an active file is created, an additional inode, linked with the active inode by the prev_snap_ino, is also pre-allocated to be used as the inode of the following snapshot image. When the next snapshot is taken, there is no need to allocate and to replicate the inode of its active file. Only thing to be performed at that time is to adjust two pointers, prev_core_snap and next_core_snap, and to setup the bitmap value of the extent to denote the sharing of the data blocks. The Open ROW Snapshot provides a snapshot daemon that periodically wakes up and issues a snapshot system call to check the state of each snapshot image.

\subsection{Snapshot Procedure}

The Open ROW Snapshot assigns a bitmap value to each extent structure to manage the sharing of the data blocks, as shown in Fig. 2(a). If the bitmap value is set to 0 , it then means that the data blocks of the extent can not be modified because those blocks must have been shared with other files. Otherwise, the 
blocks of the extent can be modified. Figure 2/(a) shows an active file using the extent storage structure. The file is composed of five data blocks, B0 through B4, and its extent includes three components: the starting block number, block count and bitmap value. Because there is no snapshot taken yet, the bitmap value is set to 1 , meaning that no other file currently shares the data blocks belonging to this file. Figure 2(b) describes the steps involved in taking the first snapshot. The file located at the left side in Fig. 2(b) denotes an active file and the file at the right side denotes its point-in-time snapshot image.

As can be seen in Fig. 2(b), the bitmap value of the active file is changed from 1 to 0 at the time of taking the first snapshot image because the data blocks of the active file are shared with those of the first snapshot. Changing a bitmap value has a significant performance impact on data modification or deletion because, by checking the current value of the bitmap, we can determine if the corresponding data block enables to be updated or the new blocks must be allocated to get the new data values.

Figure 2(c) describes the steps involved in the first update occurred after the first snapshot image was taken. In Fig. 2(c), the update requires two blocks, B3 and B4, to be modified. Since these two blocks are shared between the active file and the first snapshot image, two new blocks, B5 and B6, are allocated and then the update is performed on these two new blocks, without touching the two original blocks, B3 and B4. Also, to reflect the new block allocation for the update, an additional extent is created and its bitmap value is initially set to 1 because no other file currently shares these new data blocks with the original file.

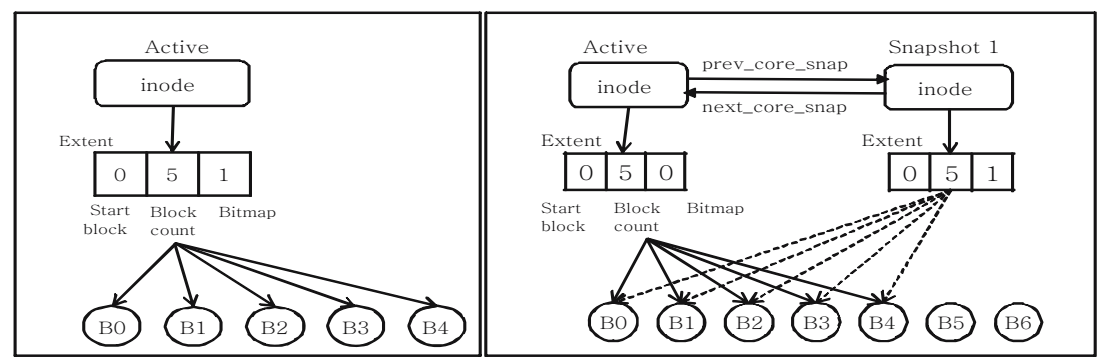

(a) An active file

(b) Take a first snapshot image

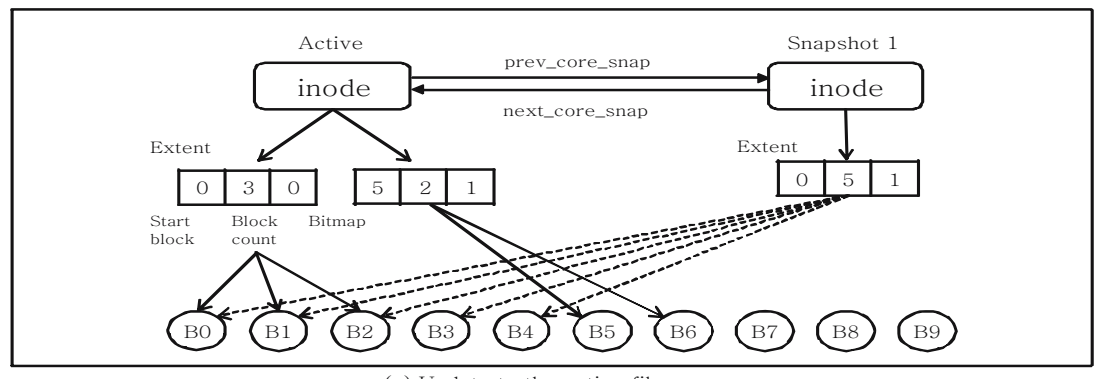

(c) Update to the active file

Fig. 2. Snapshot procedure in Open ROW Snapshot 


\subsection{Spatial Optimizations}

In order to minimize I/O processing overhead to be occurred while a snapshot is taken, the Open ROW Snapshot pre-allocates the inode for the next snapshot and duplicates all the data block addresses to the pre-allocated inode. Furthermore, to maintain the snapshot image groups in a small-sized disk section, the snapshot daemon is periodically waken up to make sure that all the snapshot images are in a consistent state. When the snapshot daemon finds a snapshot image that has been corrupted or an image that has been backup-ed to other disk, the daemon eliminates the snapshot by unlinking it from the snapshot history.

The Open ROW Snapshot provides a snapshot spatial algorithm in which any snapshot image linked at the middle of the history can efficiently be deleted by checking the sharing of the data blocks. Figures 3(a) and (b) show the steps involved in the snapshot spatial algorithm to eliminate a snapshot image, snapshot2. Figure 3(a) describes a snapshot overview before eliminating a corrupted snapshot image, snapshot2. In this Figure, the first snapshot image, snapshot1, includes an extent denoting that four data blocks, B0 through B3, are allocated to this image. The second snapshot image, snapshot2, inherits four data blocks from snapshot1, while changing the bitmap value from 1 to 0 because those data blocks are shared between these two files. It is noted that when snapshot 2 was an active file, there existed a write operation requiring three new

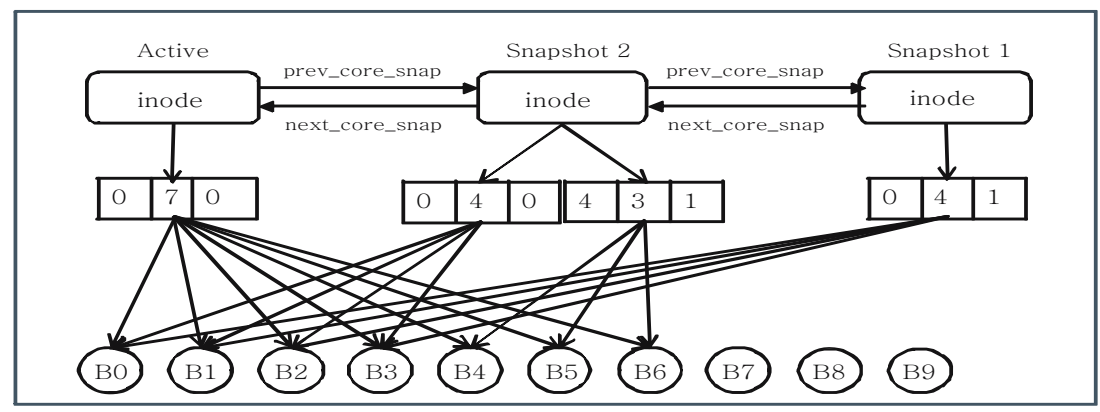

(a) Before eliminating snapshot2

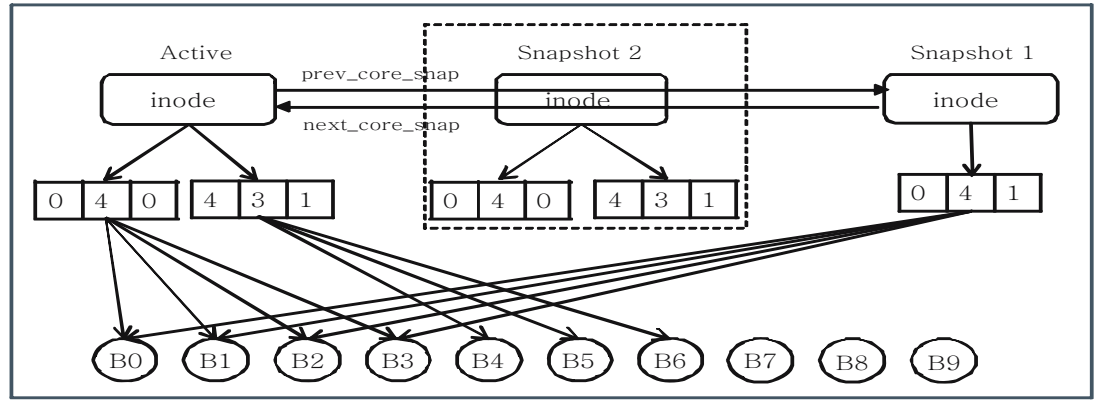

(b) After eliminating snapshot2

Fig. 3. Snapshot elimination process 
data blocks, B4 through B6, to be allocated. Therefore, the Open ROW Snapshot assigns an extent to the inode of snapshot 2 while setting its bitmap value to 1 because no sharing happened yet to those new blocks.

When the second snapshot was taken, the active file contains these seven data blocks, B0 through B6. Suppose that the snapshot daemon finds the second snapshot image, snapshot2, was corrupted. The elimination process occurred in the snapshot spatial algorithm requires the daemon to traverse prev_core_snap and next_core_snap pointers to determine if the data blocks belonging to the file to be deleted can be deallocated. The snapshot spatial algorithm to eliminate a corrupted image works as follows.

- In case that the bitmap value of an extent is of 0 . It denotes that the blocks belonging to this extent can not be modified because those blocks are shared with other file linked by the prev_core_snap pointer.

- In case that the bitmap value of an extent is of 1 . It denotes that the blocks belonging to this extent is not shared with the preceding snapshot images. However, these data blocks can be shared with other following snapshot images connected to by the next_core_snap pointer. Therefore, the traversal through the next_core_snap pointer is needed to execute.

In order to eliminate snapshot2, the bitmap value of all the extents of snapshot2 should be checked. As can be seen in Fig. 3(b), snapshot2 has two extents to manage the data blocks associated. Since the bitmap value of the first extent is set to 0 , the corresponding data blocks can not be deallocated, and thus the snapshot spatial algorithm simply unlinks the pointers to those data blocks. The bitmap value of the second extent is of 1 , therefore the preceding snapshot images connected to by the prev_core_snap pointer with snapshot 2 has not shared the data blocks with snapshot2. However, since these data blocks can be shared with the following snapshot images or the active file, before de-allocating the data blocks, the spatial algorithm should take into account the bitmap value of the active file labeled as active in Fig. 3(b). Because the active file includes the data blocks being shared with snapshot2, the snapshot spatial algorithm splits the extent into two parts to separate the data blocks. The bitmap value of the first split extent is set to 0 because, even though snapshot 2 is eliminated, the associated data blocks, B0 through B3, are still shared with the first snapshot image, snapshot1. On the other hand, the bitmap value of the second split extent is set to 1 , because, after eliminating snapshot2 image, no other file shares the associated data blocks, B4 through B6, with the active file.

\section{Performance Evaluation}

We obtained all performance results on the Linux cluster at Sejong university. We installed the Open ROW Snapshot on top of XFS using the extent-based allocation policy and produced the performance results. Figures 4 through 9 compare the performance measurements of the Open ROW Snapshot structure and the LVM snapshot. Figures 4 and 5 show the results measured using small 

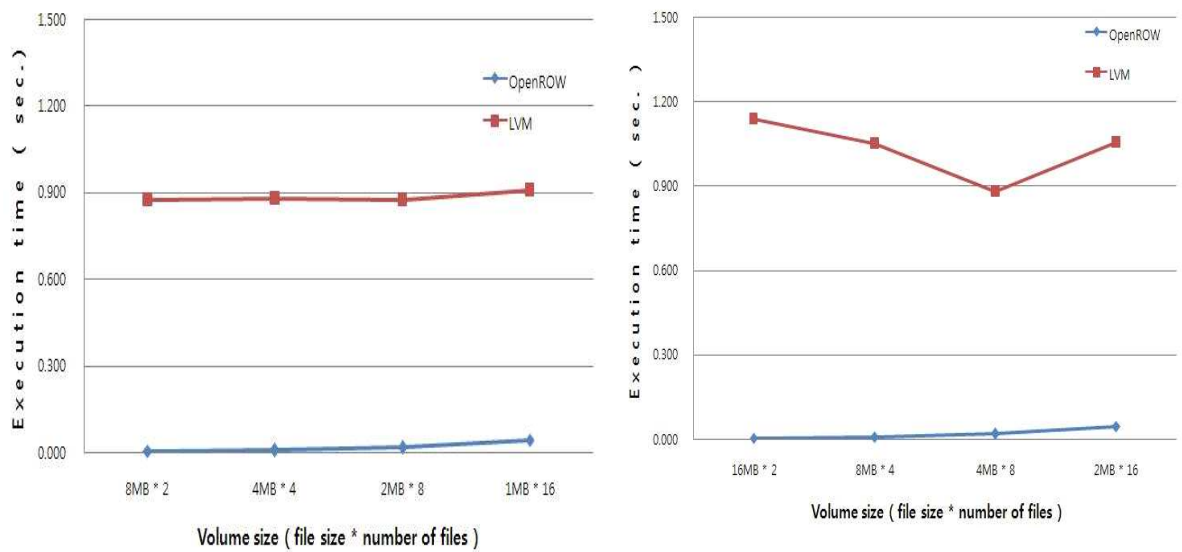

Fig. 4. Snapshot execution time using the Fig. 5. Snapshot execution time using the small size of files $(1 \mathrm{MB}-8 \mathrm{MB})$ small size of files (2MB - 16MB)
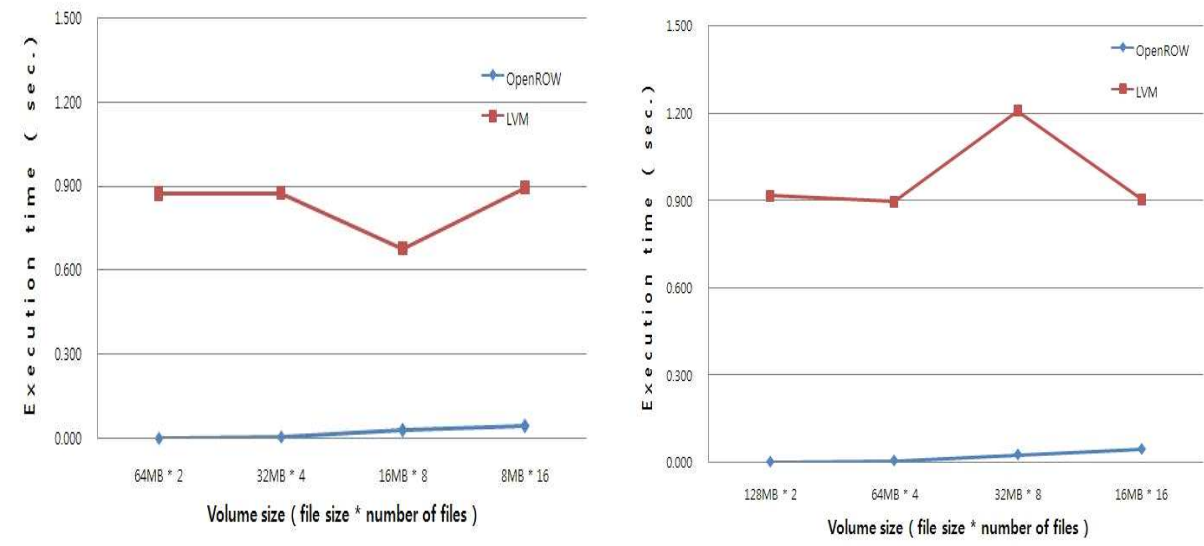

Fig. 6. Snapshot execution time using the Fig. 7. Snapshot execution time using the intermediate size of files ( $8 \mathrm{MB}-64 \mathrm{MB}) \quad$ intermediate size of files (16MB - 128MB)

files. At each experiment, we calculated the volume size to be the file size multiplied by the number of files. With the LVM snapshot, no matter which files need to be taken pictures, the total volume size should be duplicated, therefore, the time for taking a snapshot image is quite high. On the other hand, the Open ROW Snapshot only takes duplicated images for the files that are not either backup-ed, or corrupted. Furthermore, in order to minimize the I/O processing overhead, we used the pre-allocated inode for a following snapshot image. These optimizations help to produce better performance in the Open ROW Snapshot than in the LVM snapshot. Quite similar results are obtained with the 


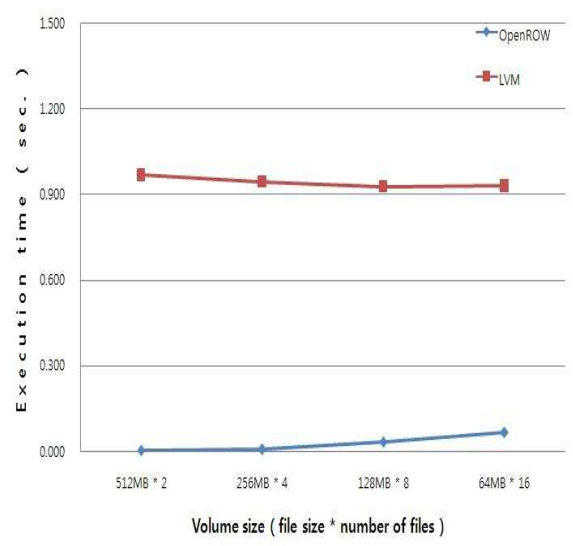

Fig. 8. Snapshot execution time using the large size of files $(64 \mathrm{MB}-512 \mathrm{MB})$

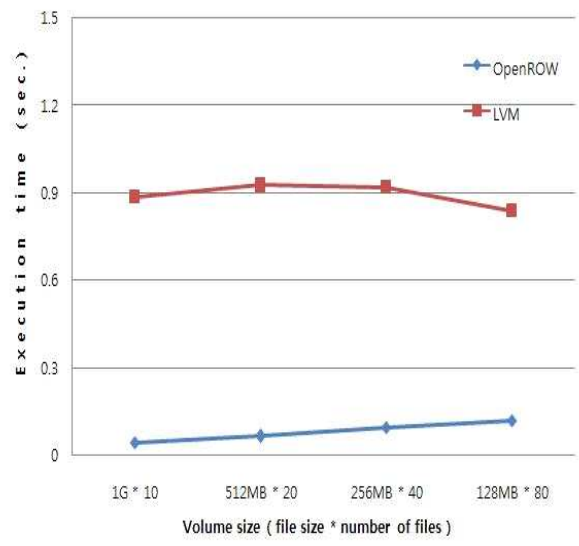

Fig. 9. Snapshot execution time using the large size of files $(128 \mathrm{MB}-1 \mathrm{~GB})$

intermediate file size, as shown in Fig. 6 and Fig. 7, and with the large file size, as shown in Fig. 8 and Fig. 9 .

\section{Conclusion}

In this paper, we presented the Open ROW Snapshot that combined the good features of both file system-based method and ROW approach. The Open ROW Snapshot was designed to minimize I/O processing overhead occurred between successive snapshot images, to provide a wide range of portability by supporting both the extent-unit and block-unit allocation policies, and to provide a capability of managing disk space for snapshots in a consistent size of disk section. We presented the performance results obtained from the Linux cluster at Sejong university. With the LVM snapshot, no matter which files need to be taken pictures, the total volume size should be duplicated, therefore, the time for taking a snapshot image is quite high. On the other hand, the Open ROW Snapshot only takes duplicated images for the files that are not either backup-ed, or corrupted. This optimization helps to produce better performance in the Open ROW Snapshot than in the LVM snapshot. In the future work, we will justify and improve the performance of the Open ROW Snapshot by doing more experiments.

\section{References}

1. Sweeney, A., Doucette, D., Hu, W., Anderson, C., Nishimoto, M., Peck, G.: Scalability in the XFS File system. In: USENIX 1996: Annual Technical Conference (1996)

2. Mostek, J., Earl, W., Koren, D.: Porting the SGI XFS File System. In: Linux 6th Linux Kongress: The Linux Storage Management Workshop, LSMW (1999) 
3. Peterson, Z.N.J., Burns, R.C.: Ext3cow: The design, implementation, and analysis of metadata for a timeshifting file system. Technical report, Department of Computer Science, The Johns Hopkins University (2003)

4. Peterson, Z.N.J., Burns, R.C.: Ext3cow: A Time-Shifting File System for Regulatory Compliance. ACM Transactions on Storage 1(2), 190-212 (2005)

5. Shim, S., Lee, W., Park, C.: An Efficient Snapshot Technique for Ext3 File System in Linux 2.6. Technical report, Pohang University of Science And Technology

6. Americal Megatrends. Inc., AMI Snapshot Thechnology, Technical report (2005)

7. Chapman, D., Merrill, J.: Open systems SnapVault. Technical Report 3466, Network Appliance (2006)

8. Mary, B., Perterson, D.: Integrating Network Appliance Snapshot and SnapRestore with Veritas Netbackup in an Oracle Backup Environment. Technical Report 3394, Network Appliance (2006)

9. Patterson, H., Manley, S., Federwisch, M., Hitz, D., Kleiman, S., Owara, S.: SnapMirror: File System Based Asynchronous Mirroring for Disaster Recovery. In: Proceedings of the FAST 2002 Conference on File and Storage Technologies, pp. 28-30 (2002)

10. SuSE Inc., The Logical Volume Manager (LVM), Technical report (2002)

11. Piernas, J., Cortes, T., Garcia, J.: DualFS: a New Journaling File System without Meta -Data Duplication. In: Proceedings of the 2002 International Conference on Supercomputing (2002)

12. Seltzer, M., Bostic, K., McKusick, M.K., Staelin, C.: An Implementation of a LogStructured File System for UNIX. In: USENIX Annual Technical Conference (1993)

13. Santry, D., Feeley, M., Hutchinson, N., Veitch, A.: Elephant: The File System that Never Forgets. In: Proceedings of IEEE Hot Topics in Operating Systems (1999) 